\title{
Kinematics and Dynamics of a Tensegrity-Based Water Wave Energy Harvester
}

\author{
Min Lin, ${ }^{1}$ Tuanjie Li, ${ }^{1}$ and Zhifei $\mathbf{J i}^{2}$ \\ ${ }^{1}$ School of Electro-Mechanical Engineering, Xidian University, Xian 710071, China \\ ${ }^{2}$ College of Mechanical and Energy Engineering, Jimei University, Xiamen 361021, China \\ Correspondence should be addressed to Zhifei Ji; zfi118@163.com
}

Received 5 January 2016; Accepted 22 May 2016

Academic Editor: Shahram Payandeh

Copyright (c) 2016 Min Lin et al. This is an open access article distributed under the Creative Commons Attribution License, which permits unrestricted use, distribution, and reproduction in any medium, provided the original work is properly cited.

\begin{abstract}
A tensegrity-based water wave energy harvester is proposed. The direct and inverse kinematic problems are investigated by using a geometric method. Afterwards, the singularities and workspaces are discussed. Then, the Lagrangian method was used to develop the dynamic model considering the interaction between the harvester and water waves. The results indicate that the proposed harvester allows harvesting $13.59 \%$ more energy than a conventional heaving system. Therefore, tensegrity systems can be viewed as one alternative solution to conventional water wave energy harvesting systems.
\end{abstract}

\section{Introduction}

Tensegrity systems are formed by a combination of rigid elements (struts) under compression and elastic elements (cables or springs) under tension. The use of cables or springs as tensile components leads to an important reduction in the weight of the systems. Due to this attractive nature, tensegrity systems have been proposed to be used in many disciplines. Moreover, a detailed description of the history of tensegrity systems is provided in $[1,2]$.

The first research work that deals with tensegrity systems was completed by Calladine [3]. Since then, tensegrity systems have been rapidly applied as structures in the architectural context. A tensegrity dome was proposed by Pellegrino [4]. Some design methods for tensegrity domes are proposed by $\mathrm{Fu}$ [5]. Afterwards, tensegrity structures have been also proposed to be served as bridges [6-9]. Moreover, the use of cables or springs in tensegrities allows them to be deployable $[10,11]$. Due to this nature, some research works are found towards their use as antennas $[12,13]$. For static applications, the subject of form-finding of tensegrities has attracted the attention of several researchers $[14,15]$. Moreover, a review of form-finding methods was provided by Tibert and Pellegrino [16]. The basic issues about the statics of tensegrity structures were reviewed by Juan and Tur [17].
From an engineering point of view, tensegrities are a special class of structures whose components may simultaneously perform the purposes of structural force, actuation, sense, and feedback control. For such kind of structure, pulleys or other kinds of actuators may stretch/shorten some of the constituting components in order to substantially change their forms with a little variation of the structure's energy. Ingber [18] has demonstrated that tensegrity structures are very similar to cytoskeleton structures of unicellular organisms. Afterwards, the cellular tensegrity model is used to understand the cell structure, biological networks, and mechanoregulation $[19,20]$. Tensegrity structures are also very similar to muscle-skeleton structures of high efficiency land animals whose speeds can reach up to $60 \mathrm{mph}$. The muscle-skeleton systems of these beings are composed of only tensional and compressional components. They thus have the ability to run with high speed [21].

Another interesting application of tensegrities is their development for use as mechanisms. Oppenheim and Williams [22] were the first to consider the actuation of tensegrity systems by modifying the lengths of their components in order to obtain tensegrity mechanisms. Afterwards, several mechanisms based on tensegrity systems were proposed, such as a flight simulator [23], a space telescope 


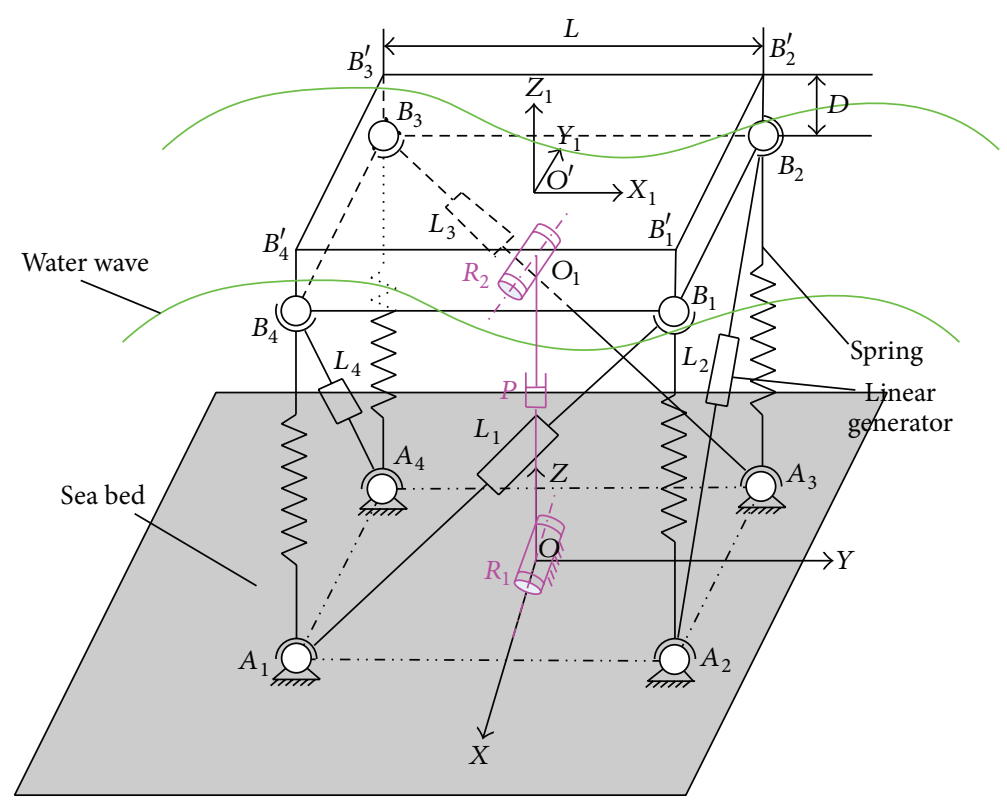

FIGURE 1: A tensegrity-based water wave energy harvester.

[24], and a tensegrity walking robot [25-27]. For tensegrity mechanisms, an interesting topic named tensegrity parallel mechanism has been proposed recently. The concept of tensegrity parallel mechanism was introduced by Marshall [28]. Then, Shekarforoush et al. [29] presented the statics of a 3-3 tensegrity parallel mechanism. Afterwards, Crane III et al. [30] proposed a planar tensegrity parallel mechanism and completed its equilibrium analysis. Tensegrity systems have been identified as one of three main research trends in mechanisms and robotics for the second decade of the 21st century [31]. However, just a few references have stated the possibility of using tensegrity systems as water wave energy harvesters. Scruggs and Skelton [32] made a preliminary investigation on the potential use of controlled tensegrity structures to harvest energy. Sunny et al. [33] studied the feasibility of harvesting energy using polyvinylidene fluoride patches mounted on vibrating prestressed membrane. Vasquez et al. [34] stated the possibility of using a planar tensegrity mechanism in ocean applications. This application is attractive since it can play an important role in the expansion of clean energy technologies that help the world's sustainable development.

This work presents the analysis of a tensegrity-based water wave energy harvester. Since this is the first stage for the development of a new application for tensegrity systems, a simplified linear model of sea waves was used to analyze the proposed harvester. The analytical solutions to the direct and inverse kinematic problems are found using a geometric method. Based on the obtained relationships between the input and output variables, the singular configurations have been discussed. The workspaces of the proposed mechanism have subsequently been computed. Afterwards, the dynamics were investigated. Finally, the energy harvesting capabilities of the tensegrity-based harvester are compared with a conventional heaving system.

\section{Geometry of the Water Wave Energy Harvester}

A diagram of the tensegrity-based water wave energy harvester is shown in Figure 1. It consists of a float, four springs, four linear generators, and one kinematic chain. The linear generators are joining node pairs $A_{i} B_{i}(i=1,2,3,4)$ while the springs are joining node pairs $A_{1} B_{4}, A_{2} B_{1}, A_{3} B_{2}$, and $A_{4} B_{3}$. The float of height $D$ is denoted by $B_{1} B_{2} B_{3} B_{4}$. This harvester is obtained from a square tensegrity parallel prism [10] by connecting the top of the latter to a float.

From Figure 1, it can be seen that the sides of the squares formed by nodes $A_{1} A_{2} A_{3} A_{4}, B_{1} B_{2} B_{3} B_{4}$, and $B_{1}^{\prime} B_{2}^{\prime} B_{3}^{\prime} B_{4}^{\prime}$ have the same length $L$. Moreover, the length of the linear generator joining node pairs $A_{i} B_{i}$ is denoted by $L_{i}$. As illustrated in Figure 1 , the springs and the linear generators are connected to the float and the sea bed at nodes $A_{i}$ and $B_{i}$ by spherical joints without friction. The sea bed is considered to be parallel to the horizontal plane. A fixed reference frame $A(X, Y, Z)$ is located at the center of the square $A_{1} A_{2} A_{3} A_{4}$ with its $X$ axis parallel to the line joining nodes $A_{4}$ and $A_{1}$ and its $Z$ axis perpendicular to the sea bed, while a moving reference frame $B\left(X_{1}, Y_{1}, Z_{1}\right)$ is located at the mass center of the float with its $X_{1}$ axis parallel to the line joining nodes $B_{4}$ and $B_{1}$ and its $Z_{1}$ axis perpendicular to the plane formed by nodes $B_{1}, B_{2}, B_{3}$, and $B_{4}$. Moreover, the vectors specifying the positions of nodes $A_{i}$ and $B_{i}$ in the fixed reference frame are defined as ${ }^{A} \mathbf{a}_{i}$ and ${ }^{A} \mathbf{b}_{i}$, respectively. Also, the vectors specifying the positions of nodes $B_{i}$ in the moving reference frame are defined as ${ }^{B} \mathbf{b}_{i}$.

In order to obtain an appropriate kinematic model of the harvester, the following hypotheses are made:

(i) The springs are linear with stiffness $K$ and lengths $l_{j}(j=1,2,3,4)$ and all the springs have the same free length $L_{0}$. 
(ii) The water waves are traveling along the $Y$ axis.

In Figure 1, a passive kinematic chain denoted by $R_{1} P R_{2}$ is used to connect nodes $O$ and $O_{1}$. Nodes $O$ and $O_{1}$ represent the centers of the squares $A_{1} A_{2} A_{3} A_{4}$ and $B_{1} B_{2} B_{3} B_{4}$, respectively. Considering the constraints introduced by this kinematic chain, the possible movements of the float driven by water waves are rotations about the $X$ axis and translations along the $Y$ and $Z$ axes. Therefore, the harvester has three degrees of freedom.

The Cartesian coordinates of the mass center of the float in the fixed reference frame are defined as $(x, y, z)$. From Figure 1, it can be seen that $x=0$ is always satisfied. Moreover, the angle $\theta$ is used to specify the rotation of the float about $X$ axis. Meanwhile, the range of $\theta$ is assumed to be $[-\pi / 2 \pi / 2]$. The variables $y, z$, and $\theta$ are driven by the water waves. As a consequence, they are thus chosen as the inputs of the system. Furthermore, only three of the four linear generators' lengths are independent. For this reason, the lengths of the generators joining nodes $A_{1} B_{1}, A_{2} B_{2}$, and $A_{3} B_{3}$ are chosen as the outputs of the system. It follows that the harvester's output vector is $\mathbf{O}=\left[L_{1}, L_{2}, L_{3}\right]^{T}$ while its input vector is $\mathbf{I}=[y, z, \theta]^{T}$.

\section{Kinematic Analysis}

For the harvester, the linear generators are used to convert wave motion cleanly into electricity. Generally, the efficiency of electricity generation of the system is highly dependent on the motions of linear generators. To provide great insight into the kinematics of the harvester, the relationship between the input and output vectors is developed in this section.

3.1. Direct Kinematic Analysis. The direct kinematic analysis consists in computing the output vector $\mathbf{O}$ for the given input vector I. According to [35], the most convenient approach to set an algebraic equation system for kinematic problem of a parallel mechanism is to use the rotation matrix parameters and the position vector of the moving platform. This approach is used in this work to deal with the kinematic problems of the harvester. The position and orientation of the float are described by the position vector $\mathbf{P}=\mathbf{O O}^{\prime}=$ $[0, y, z]^{T}$ and the rotation matrix ${ }^{A} \mathbf{R}_{B}$ with respect to the fixed reference frame. From Figure 1, it can be seen that the rotation matrix ${ }^{A} \mathbf{R}_{B}$ can be defined by rotating the moving reference frame $\pi / 2$ about $Z_{1}$ axis followed by $\theta$ about $Y_{1}$ axis. ${ }^{A} \mathbf{R}_{B}$ thus takes the following form:

$$
{ }^{A} \mathbf{R}_{B}=\left[\begin{array}{ccc}
0 & -1 & 0 \\
\cos \theta & 0 & \sin \theta \\
-\sin \theta & 0 & \cos \theta
\end{array}\right]
$$

Then, the position vectors of points $B_{i}(i=1,2,3,4)$ with respect to the fixed reference frame can be obtained:

$$
{ }^{A} \mathbf{b}_{i}=\mathbf{P}+{ }^{A} \mathbf{R}_{B}{ }^{B} \mathbf{b}_{i}, \quad i=1,2,3,4 .
$$

The vectors specifying the positions of nodes $B_{i}$ in the moving reference frame can be easily derived:

$$
\begin{aligned}
& { }_{\mathbf{b}_{1}}^{B^{\prime}}=\left[\begin{array}{c}
\frac{L}{2} \\
-\frac{L}{2} \\
-\frac{D}{2}
\end{array}\right], \\
& { }^{B} \mathbf{b}_{2}=\left[\begin{array}{c}
\frac{L}{2} \\
\frac{L}{2} \\
-\frac{D}{2}
\end{array}\right], \\
& { }_{\mathbf{b}_{3}}=\left[\begin{array}{c}
\frac{L}{2} \\
-\frac{L}{2} \\
-\frac{D}{2}
\end{array}\right], \\
& { }_{\mathbf{b}_{4}}=\left[\begin{array}{c}
\frac{L}{2} \\
-\frac{L}{2} \\
-\frac{D}{2}
\end{array}\right] .
\end{aligned}
$$

Substituting (3) into (2), we have

$$
\begin{aligned}
& { }_{\mathbf{b}_{1}=}=\left[\begin{array}{c}
\frac{L}{2} \\
y+\frac{L}{2} \cos \theta-\frac{D}{2} \sin \theta \\
z-\frac{D}{2} \cos \theta-\frac{L}{2} \sin \theta
\end{array}\right], \\
& { }_{\mathbf{b}_{2}}=\left[\begin{array}{c}
y+\frac{L}{2} \cos \theta-\frac{D}{2} \sin \theta \\
z-\frac{D}{2} \cos \theta-\frac{L}{2} \sin \theta
\end{array}\right], \\
& \left.{ }_{\mathbf{b}_{3}=} \begin{array}{c}
-\frac{L}{2} \\
y-\frac{L}{2} \cos \theta-\frac{D}{2} \sin \theta \\
z-\frac{D}{2} \cos \theta+\frac{L}{2} \sin \theta
\end{array}\right], \\
& { }_{\mathbf{b}_{4}}=\left[\begin{array}{c}
L \\
y-\frac{L}{2} \cos \theta-\frac{D}{2} \sin \theta \\
z-\frac{D}{2} \cos \theta+\frac{L}{2} \sin \theta
\end{array}\right] .
\end{aligned}
$$


From Figure 1, it can also be seen that ${ }^{A} \mathbf{a}_{1}=$ $[L / 2,-L / 2,0]^{T},{ }^{A} \mathbf{a}_{2}=[L / 2, L / 2,0]^{T},{ }^{A} \mathbf{a}_{3}=[-L / 2, L / 2,0]^{T}$, and ${ }^{A} \mathbf{a}_{4}=[-L / 2,-L / 2,0]^{T}$. With the position vectors of points $A_{i}$ and $B_{i}$ now known, the vector equation of the $i$ th linear generator can be written as

$$
\mathbf{L}_{i}={ }^{A} \mathbf{b}_{i}-{ }^{A} \mathbf{a}_{i}, \quad i=1,2,3,4 .
$$

By using (5), the solution to the direct kinematic problem is found as follows:

$$
\begin{aligned}
L_{1} & =\left[\left(z-\frac{D}{2} \cos \theta-\frac{L}{2} \sin \theta\right)^{2}\right. \\
& \left.+\left(y+\frac{L}{2} \cos \theta-\frac{D}{2} \sin \theta+\frac{L}{2}\right)^{2}\right]^{1 / 2}, \\
L_{2} & =\left[\left(z-\frac{D}{2} \cos \theta-\frac{L}{2} \sin \theta\right)^{2}\right. \\
& \left.+\left(y+\frac{L}{2} \cos \theta-\frac{D}{2} \sin \theta-\frac{L}{2}\right)^{2}+L^{2}\right]^{1 / 2}, \\
L_{3} & =\left[\left(z-\frac{D}{2} \cos \theta+\frac{L}{2} \sin \theta\right)^{2}\right. \\
& \left.+\left(y-\frac{L}{2} \cos \theta-\frac{D}{2} \sin \theta-\frac{L}{2}\right)^{2}\right]^{1 / 2} .
\end{aligned}
$$

Here, for the latter use, the length of the linear generator joining nodes $A_{4} B_{4}$ is also presented:

$$
\begin{aligned}
L_{4} & =\left[\left(z-\frac{D}{2} \cos \theta+\frac{L}{2} \sin \theta\right)^{2}\right. \\
& \left.+\left(y-\frac{L}{2} \cos \theta-\frac{D}{2} \sin \theta+\frac{L}{2}\right)^{2}+L^{2}\right]^{1 / 2} .
\end{aligned}
$$

3.2. Inverse Kinematic Analysis. The inverse kinematic problem corresponds to the computation of the input vector I for the given output vector $\mathbf{O}$. The solution to this problem can be found by solving (6)-(8) for the input variables $y, z$, and $\theta$. Subtracting the square of (7) from that of (6) yields

$$
2 L y+L^{2} \cos \theta-L^{2}-L_{1}^{2}+L_{2}^{2}-D L \sin \theta=0 .
$$

Subtracting the square of (8) from that of (6), we obtain

$$
2 L y(\cos \theta+1)-D L \sin \theta-L_{1}^{2}+L_{3}^{2}-2 L z \sin \theta=0 .
$$

From (10) and (11), the following expressions can be derived:

$$
\begin{aligned}
y= & \frac{1}{2 L}\left(L^{2}+L_{1}^{2}-L_{2}^{2}-L^{2} \cos \theta+D L \sin \theta\right), \\
z= & \frac{1}{2 L \sin \theta}\left[L^{2} \sin ^{2} \theta+D L \sin \theta \cos \theta\right. \\
& \left.+\left(L_{1}^{2}-L_{2}^{2}\right) \cos \theta-L_{2}^{2}+L_{3}^{2}\right] .
\end{aligned}
$$

By substituting (12) into (6), the following equation is obtained:

$$
\begin{aligned}
4\left(L^{2}-\right. & \left.L_{2}^{2}\right) L^{2} \cos ^{2} \theta \\
- & {\left[\left(L_{1}^{2}-L_{2}^{2}\right)^{2}+\left(L_{2}^{2}-L_{3}^{2}\right)^{2}-\left(L_{1}^{2}-L_{3}^{2}\right)^{2}\right] \cos \theta } \\
& -4 L^{2}\left(L^{2}-L_{2}^{2}\right)-\left(L_{1}^{2}-L_{2}^{2}\right)^{2}-\left(L_{2}^{2}-L_{3}^{2}\right)^{2}=0 .
\end{aligned}
$$

Because of the range imposed on $\theta$, four solutions for $\theta$ can be arrived at by solving (13). Furthermore, by substituting these results into (12), the solutions to the inverse kinematic problem are found.

\section{Singularity Analysis}

4.1. Jacobian Matrix. The Jacobian matrix of the harvester is defined as the relationships between a set of infinitesimal changes of its input vectors and the corresponding infinitesimal changes of its output vectors. The Jacobian matrix, J, relates $\delta \mathbf{I}$ to $\delta \mathbf{O}$ such that $\delta \mathbf{O}=\mathbf{J} \delta \mathbf{I}$. $\mathbf{J}$ can be rewritten in terms of matrices $\mathbf{C}$ and $\mathbf{D}$ such that $\mathbf{C} \delta \mathbf{O}=\mathbf{D} \delta \mathbf{I}$. From (6)(8), the elements of $\mathbf{C}$ and $\mathbf{D}$ can be computed and written in terms of the input variables as follows:

$$
\begin{aligned}
C_{11} & =\left[\left(z-\frac{D}{2} \cos \theta-\frac{L}{2} \sin \theta\right)^{2}\right. \\
+ & \left.\left(y+\frac{L}{2} \cos \theta-\frac{D}{2} \sin \theta+\frac{L}{2}\right)^{2}\right]^{1 / 2}, \\
C_{22} & =\left[\left(z-\frac{D}{2} \cos \theta-\frac{L}{2} \sin \theta\right)^{2}\right. \\
& \left.+\left(y+\frac{L}{2} \cos \theta-\frac{D}{2} \sin \theta-\frac{L}{2}\right)^{2}+L^{2}\right]^{1 / 2}, \\
C_{33} & =\left[\left(z-\frac{D}{2} \cos \theta+\frac{L}{2} \sin \theta\right)^{2}\right. \\
+ & \left.\left(y-\frac{L}{2} \cos \theta-\frac{D}{2} \sin \theta-\frac{L}{2}\right)^{2}\right]^{1 / 2}, \\
C_{12} & =C_{13}=C_{21}=C_{23}=C_{31}=C_{32}=0, \\
D_{11} & =y+\frac{L}{2} \cos \theta-\frac{D}{2} \sin \theta+\frac{L}{2}, \\
D_{12} & =D_{22}=z-\frac{D}{2} \cos \theta-\frac{L}{2} \sin \theta, \\
D_{13} & =\left(z-\frac{D}{2} \cos \theta-\frac{L}{2} \sin \theta\right)\left(\frac{D}{2} \sin \theta-\frac{L}{2} \cos \theta\right) \\
- & \left(y+\frac{L}{2} \cos \theta-\frac{D}{2} \sin \theta+\frac{L}{2}\right)\left(\frac{L}{2} \sin \theta\right. \\
- & \left.\frac{D}{2} \cos \theta\right),
\end{aligned}
$$




$$
\begin{aligned}
D_{21} & =y+\frac{L}{2} \cos \theta-\frac{D}{2} \sin \theta-\frac{L}{2}, \\
D_{31} & =y-\frac{L}{2} \cos \theta-\frac{D}{2} \sin \theta-\frac{L}{2}, \\
D_{32} & =z-\frac{D}{2} \cos \theta+\frac{L}{2} \sin \theta, \\
D_{23} & =\left(z-\frac{D}{2} \cos \theta-\frac{L}{2} \sin \theta\right)\left(\frac{D}{2} \sin \theta-\frac{L}{2} \cos \theta\right) \\
- & \left(y+\frac{L}{2} \cos \theta-\frac{D}{2} \sin \theta-\frac{L}{2}\right)\left(\frac{L}{2} \sin \theta\right. \\
+ & \left.\frac{D}{2} \cos \theta\right), \\
D_{33} & =\left(z-\frac{D}{2} \cos \theta+\frac{L}{2} \sin \theta\right)\left(\frac{D}{2} \sin \theta+\frac{L}{2} \cos \theta\right) \\
& +\left(y-\frac{L}{2} \cos \theta-\frac{D}{2} \sin \theta-\frac{L}{2}\right)\left(\frac{L}{2} \sin \theta\right. \\
& \left.-\frac{D}{2} \cos \theta\right) .
\end{aligned}
$$

For (14), it is noted that $C_{i j}$ and $D_{i j}$ are the elements located on the $i$ th line and $j$ th column of $\mathbf{C}$ and $\mathbf{D}$, respectively.

4.2. Singular Configurations. The singular configurations of the harvester consist in finding the situations where the relationships between infinitesimal changes in its input and output variables degenerate. When such a situation occurs, the harvester will gain or lose one or more degrees of freedom, thus leading to a loss of control. As a consequence, such configurations are usually avoided when possible. Generally, the singular configurations of the harvester can be obtained by setting $\operatorname{det}(\mathbf{C})=0, \operatorname{det}(\mathbf{D})=0$, or both. The determinants of $\mathbf{C}$ and $\mathbf{D}$ can be expressed as follows:

$$
\begin{aligned}
& \operatorname{det}(\mathbf{C})=\left[\left(z-\frac{D}{2} \cos \theta-\frac{L}{2} \sin \theta\right)^{2}\right. \\
& \left.+\left(y+\frac{L}{2} \cos \theta-\frac{D}{2} \sin \theta+\frac{L}{2}\right)^{2}\right]^{1 / 2} \\
& \cdot\left[\left(z-\frac{D}{2} \cos \theta-\frac{L}{2} \sin \theta\right)^{2}\right. \\
& \left.+\left(y+\frac{L}{2} \cos \theta-\frac{D}{2} \sin \theta-\frac{L}{2}\right)^{2}+L^{2}\right]^{1 / 2} \\
& \cdot\left[\left(z-\frac{D}{2} \cos \theta+\frac{L}{2} \sin \theta\right)^{2}\right. \\
& \left.+\left(y-\frac{L}{2} \cos \theta-\frac{D}{2} \sin \theta-\frac{L}{2}\right)^{2}\right]^{1 / 2}=0,
\end{aligned}
$$

$$
\begin{aligned}
& \operatorname{det}(\mathbf{D})=\frac{L^{2}}{8}[(D L-2 D y-2 L z) \sin 2 \theta \\
& \quad+\left(2 L y-2 D z-L^{2}\right) \cos 2 \theta+\left(8 z^{2}+2 D^{2}\right) \cos \theta \\
& \left.+(8 y z-4 L z+2 D L) \sin \theta+L^{2}-6 D z-2 L y\right] \\
& \quad=0 .
\end{aligned}
$$

By examining (15), it is possible to extract the expressions corresponding to singular configurations. The following is a list of these expressions as well as their descriptions with respect to the mechanism's behaviors:

$$
\text { (i) } \begin{aligned}
& {\left[\left(z-\frac{D}{2} \cos \theta-\frac{L}{2} \sin \theta\right)^{2}\right.} \\
+ & \left.\left(y+\frac{L}{2} \cos \theta-\frac{D}{2} \sin \theta+\frac{L}{2}\right)^{2}\right]^{1 / 2}=0 .
\end{aligned}
$$

(a) The length of the linear generator joining nodes $A_{1}$ and $B_{1}$ is equal to zero. Node $A_{1}$ is thus coincident with node $B_{1}$. Moreover, node $A_{4}$ is also coincident with node $B_{2}$.

(b) The movement of the float is reduced to a rotation about the axis joining nodes $A_{1}$ and $A_{4}$. When this is the case, only one variable is needed to define the system. The harvester thus loses two degrees of freedom.

(c) Infinitesimal movements of node $O^{\prime}$ in a direction perpendicular to the line joining nodes $A_{1}$ and $B_{4}$ are possible without deforming the springs and the linear generators.

(d) External forces parallel to the line $B_{1} B_{4}$ are resisted by the harvester.

$$
\text { (ii) } \begin{aligned}
& {\left[\left(z-\frac{D}{2} \cos \theta+\frac{L}{2} \sin \theta\right)^{2}\right.} \\
+ & \left.\left(y-\frac{L}{2} \cos \theta-\frac{D}{2} \sin \theta-\frac{L}{2}\right)^{2}\right]^{1 / 2}=0 .
\end{aligned}
$$

(a) The length of the linear generator joining nodes $A_{3}$ and $B_{3}$ is equal to zero. Node $A_{2}$ is coincident with node $B_{4}$.

(b) The movement of the float is reduced to a rotation about the axis joining nodes $A_{2}$ and $A_{3}$. When this case occurs, only one variable can be used to describe the rotation of the float. The harvester thus loses two degrees of freedom.

(c) Infinitesimal movements of node $O^{\prime}$ in a direction perpendicular to the line joining nodes $A_{2}$ and $B_{1}$ are possible without deforming the springs and the linear generators. 
(d) External forces parallel to the line $B_{1} B_{4}$ are resisted by the harvester.

(iii) $(D L-2 D y-2 L z) \sin 2 \theta$

$$
\begin{aligned}
& +\left(2 L y-2 D z-L^{2}\right) \cos 2 \theta+\left(8 z^{2}+2 D^{2}\right) \cos \theta \\
& +(8 y z-4 L z+2 D L) \sin \theta+L^{2}-6 D z-2 L y
\end{aligned}
$$$$
=0 \text {. }
$$

(a) Actually, it is impossible to extract the behaviors of the harvester from (18). This case corresponds to the boundaries of the input workspace and will be mapped in Section 5.2. Generally speaking, when this is the case, infinitesimal movements of the input variables along a direction perpendicular to a certain surface cannot be generated.

From (16) and (17), it can be seen that the singular configuration (i) corresponds to the situation where the length of the linear generator $A_{1} B_{1}$ is equal to zero while configuration (ii) corresponds to the situation where the length of the linear generator $A_{3} B_{3}$ is equal to zero. From an engineering point of view, the linear generators are generally limited to operate within a range of nonzero lengths. However, from the aspect of mechanism's analysis, the lengths of prismatic actuators can be set to be zero. This case belongs to one kind of the singular configurations of the proposed mechanism.

\section{Workspaces}

Since the input variables $y, z$, and $\theta$ are driven by water waves, the ranges of the input variables can be used to describe the strengths of the water waves. Moreover, the amount of the electricity produced by the harvester depends on the movements of the linear generators. The ranges of the output variables can be considered as an indicator of the efficiency of energy harvesting. In this section, the ranges of the input vectors are referred to as the input workspace while the ranges of the output vectors are referred to as the output workspace. The boundaries of the input and output workspaces usually correspond to singular configurations described in Section 4.2. From (16)-(18), it can be seen that the singular configurations are expressed in terms of the input variables. According to these expressions, the boundaries of the input workspace can be computed. Afterwards, these boundaries will be mapped from the input domain into the output domain in order to generate the output workspace.

5.1. Input Workspace. The input workspace of the harvester is a volume whose boundaries correspond to singular configurations discussed in Section 4.2. An example of such a workspace with $L=1 \mathrm{~m}$ and $D=1 \mathrm{~m}$ is shown in Figure 2 .

In Figure 2, the surface corresponding to the singular configuration (iii) is identified by surface (iii). From this figure, it can be seen that the input workspace can be divided into three parts. The first part is defined by $-1 \leq y \leq 0$ and $0 \leq \theta \leq \pi / 2$. It is bounded by surface (iii) and the planes

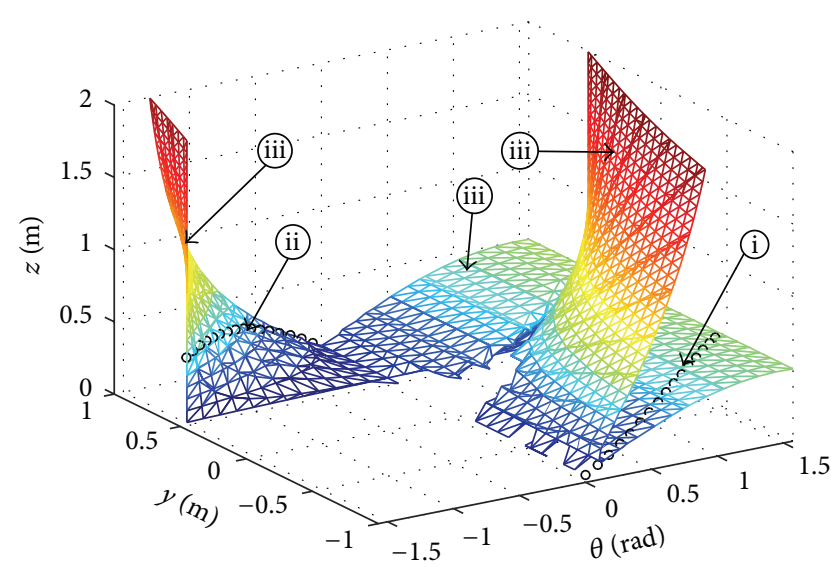

FIGURE 2: Input workspace of the harvester with $L=1 \mathrm{~m}$ and $D=$ $0.1 \mathrm{~m}$.

corresponding to $y=-1, \theta=\pi / 2$, and $z=2$. Moreover, the second part is defined by $0 \leq y \leq 1$ and $0 \leq \theta \leq \pi / 2$. It is bounded by the planes corresponding to $\theta=\pi / 2, y=1$, and surface (iii). Finally, the third part is defined by $0 \leq y \leq 1$ and $-\pi / 2 \leq \theta \leq 0$. It is bounded by the planes corresponding to $\theta=-\pi / 2, y=-1$, and surface (iii). Furthermore, from Figure 2, it can also be observed that curves (i) and (ii) correspond to the singular configurations (i) and (ii), respectively. Since the harvester will be uncontrolled when it reaches a singular configuration, the boundaries of the input workspace and the singular curves (i) and (ii) should be avoided during the use of such a harvester.

5.2. Output Workspace. In order to obtain the output workspace, the singular configurations detailed in Section 4.2 should be rewritten in terms of the output variables firstly. From (16) and (17), it can be concluded that the singular configuration (i) in the output domain corresponds to $L_{1}=0$ while the singular configuration (ii) corresponds to $L_{2}=0$. Generally speaking, by substituting the solutions to the inverse kinematic problem into (18), an expression for singular configuration (iii) in terms of the output variables can be arrived at. However, this procedure is rather tedious. Here, Bezout's method [36] was used to derive the expression corresponding to singular configuration (iii) in the output domain due to its simplicity.

Equation (13) is firstly rewritten as

$$
M_{1} \cos ^{2} \theta+M_{2} \cos \theta+M_{3}=0
$$

where

$$
\begin{aligned}
& M_{1}=4\left(L^{2}-L_{2}^{2}\right) L^{2}, \\
& M_{2}=-\left[\left(L_{1}^{2}-L_{2}^{2}\right)^{2}+\left(L_{2}^{2}-L_{3}^{2}\right)^{2}-\left(L_{1}^{2}-L_{3}^{2}\right)^{2}\right], \\
& M_{3}=-4 L^{2}\left(L^{2}-L_{2}^{2}\right)-\left(L_{1}^{2}-L_{2}^{2}\right)^{2}-\left(L_{2}^{2}-L_{3}^{2}\right)^{2} .
\end{aligned}
$$

Moreover, by substituting (12) into (18), the following equation is obtained:

$$
N_{1} \cos ^{2} \theta+N_{2} \cos \theta+N_{3}=0,
$$




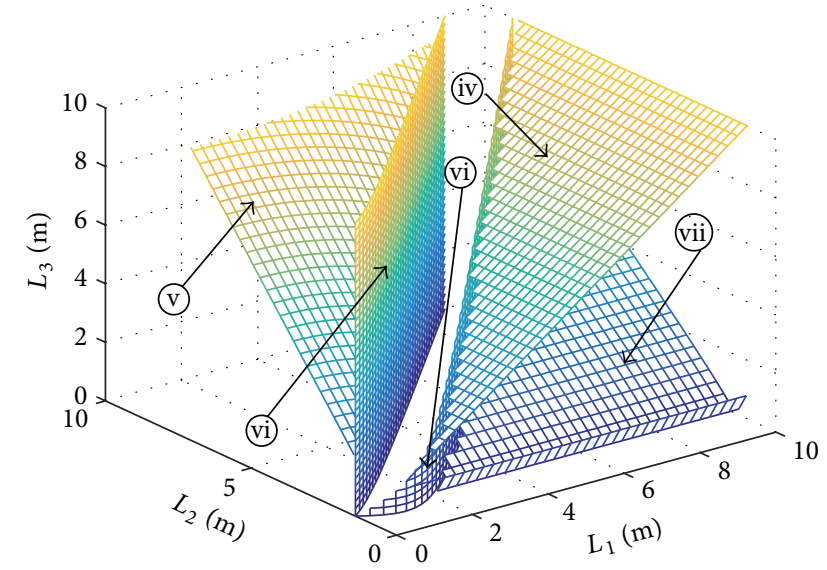

FIGURE 3: Output workspace of the water wave energy harvester with $L=1 \mathrm{~m}$ and $D=0.1 \mathrm{~m}$.

where

$$
\begin{aligned}
& N_{1}=\left(L_{1}^{2}-L_{2}^{2}\right)\left(L_{2}^{2}-L_{3}^{2}\right), \\
& N_{2}=-\left(L_{1}^{2}-L_{2}^{2}\right)^{2}-\left(L_{2}^{2}-L_{3}^{2}\right)^{2}, \\
& N_{3}=\left(L_{2}^{2}-L_{3}^{2}\right)\left(L_{2}^{2}-L_{3}^{2}\right) .
\end{aligned}
$$

It should be noted that (21) represents singular configuration (iii) expressed by $L_{1}, L_{2}, L_{3}$, and $\theta$. Moreover, (19) is used to compute $\theta$ for the given values of $L_{1}, L_{2}$, and $L_{3}$. Generally, the solutions to $\theta$ obtained by solving (21) should satisfy (19). Furthermore, both (19) and (21) can be considered as two quadratics with respect to $\cos \theta$. According to Bezout's method, the condition that (19) and (21) have a comment root for $\cos \theta$ is as follows:

$$
\left|\begin{array}{ll}
M_{1} & M_{2} \\
N_{1} & N_{2}
\end{array}\right|\left|\begin{array}{ll}
M_{2} & M_{3} \\
N_{2} & N_{3}
\end{array}\right|-\left|\begin{array}{cc}
M_{1} & M_{3} \\
N_{1} & N_{3}
\end{array}\right|^{2}=0
$$

Simplifying (23) yields

$$
\begin{aligned}
& {\left[\left(L_{1}^{2}-L_{3}^{2}\right)\left(L_{1}^{2}-2 L_{2}^{2}+L_{3}^{2}\right)\right]^{2}} \\
& \quad \cdot\left(4 L^{4}-4 L^{2} L_{2}^{2}+L_{1}^{4}-2 L_{1}^{2} L_{2}^{2}+L_{1}^{4}\right) \\
& \quad \cdot\left(4 L^{4}-4 L^{2} L_{2}^{2}+L_{2}^{4}-2 L_{2}^{2} L_{3}^{2}+L_{3}^{4}\right)=0 .
\end{aligned}
$$

Equation (24) represents the surfaces corresponding to singular configuration (iii) in the output workspace. By plotting these surfaces, the output workspace of the harvester can be obtained. An example of such plots is shown in Figure 3 with $L=1 \mathrm{~m}$ and $D=0.1 \mathrm{~m}$.

From Figure 3, it can be seen that the singular configuration (iii) determined by (24) corresponds to four surfaces (surfaces (iv)-(vii)) in the output workspace. Moreover, surfaces (iv), (v), (vi), and (vii) correspond to expressions $L_{1}-L_{3}=0, L_{1}^{2}-2 L_{2}^{2}+L_{3}^{2}=0,4 L^{4}-4 L^{2} L_{2}^{2}+L_{1}^{4}-2 L_{1}^{2} L_{2}^{2}+L_{1}^{4}=0$, and $4 L^{4}-4 L^{2} L_{2}^{2}+L_{2}^{4}-2 L_{2}^{2} L_{3}^{2}+L_{3}^{4}=0$, respectively. It can also be observed that the output workspace of the harvester can be divided into two parts. The first part is bounded by surface (v), surface (vi), plane $L_{1}=0$, and plane $L_{3}=0$ while the second part is bounded by surfaces denoted by (iv), (vi), and (vii) and planes denoted by $L_{2}=0$ and $L_{1}=10$. This output workspace should be considered during the use and design of such a harvester.

It is noted that the forward and inverse kinematics, Jacobian matrix, and workspaces should be considered when such harvester is being designed. Moreover, when the harvester is put to use, the singular configurations should be avoided. The kinematics and Jacobian matrix are used to find the singular configurations.

\section{Dynamic Analysis}

The efficiency of the water wave harvesting is highly dependent on the dynamics of the harvester. Therefore, it is of utmost importance to research the dynamics of the harvester. In this section, the dynamic model of the harvester is developed. Furthermore, in order to compare the efficiency of a conventional heaving system with that of the proposed harvester, the dynamic model of the conventional heaving system is firstly introduced. Before introducing the dynamic models of the two systems, it is assumed that the linear water waves are applied on the two systems.

6.1. Dynamic Model of a Conventional Heaving System. A diagram of the conventional heaving wave energy harvester [37] composed of a float, a bar magnet, and a battery is shown in Figure 4. In order to compare the efficiency of the conventional heaving system with the proposed harvester, the floats of both systems are assumed to have the same size. Moreover, in this paper, the weight of the bar magnet was neglected.

According to [38], the motion equation of the float, driven by linear water waves, in a conventional heaving system is given by

$$
\begin{aligned}
(m+ & \left.a_{w z}\right) \frac{d^{2} z}{d t^{2}}+\left(b_{r z}+b_{v z}+b_{p z}\right) \frac{d z}{d t} \\
& +\left(\rho g A_{w p}+N k_{s}\right) z=F_{z 0} \cos \left(\omega t+\alpha_{z}\right) .
\end{aligned}
$$

The coefficients in (25) are given as follows:

$m$ is the mass of the float.

$a_{w z}$ is the added mass.

$b_{r z}$ is the damping coefficient.

$b_{v z}$ is the viscous damping coefficient.

$b_{p z}$ is the power take-off coefficient.

$A_{w p}$ is the waterplane area when the body is at rest.

$\rho$ is the density of seawater.

$g$ is the acceleration due to gravity.

$k_{s}$ is the spring constant of mooring lines and $N$ is the number of lines (mooring restoring force). 


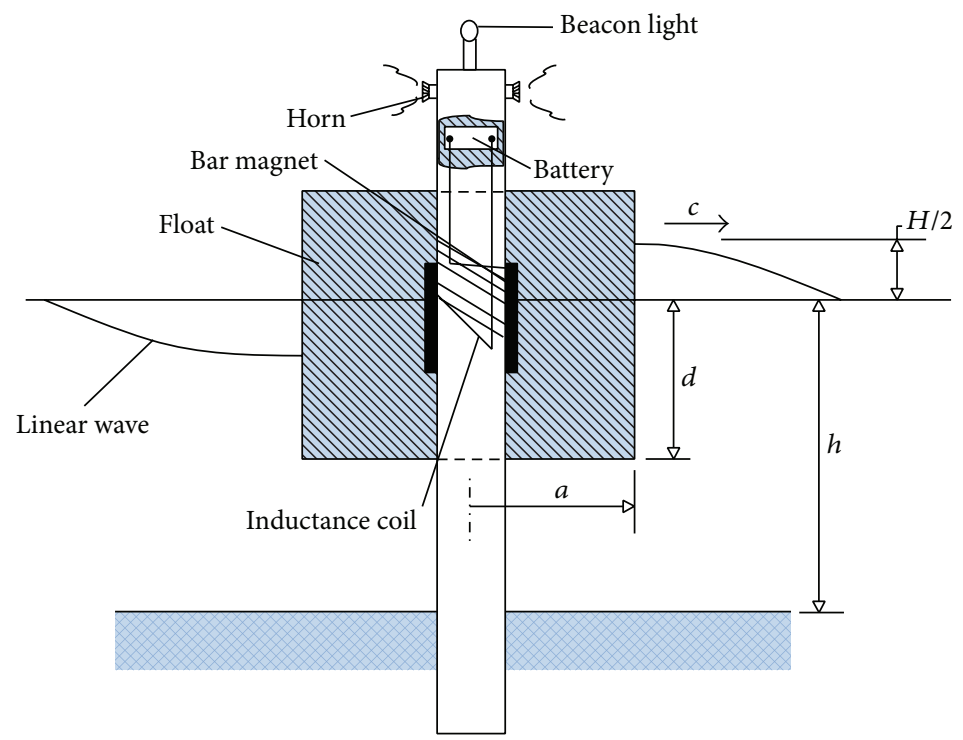

FIGURE 4: A conventional heaving wave energy harvester [37].

$F_{z 0}$ is the water-induced vertical force amplitude and $\omega=2 \pi / T$ is the circular wave frequency ( $T$ is the wave period).

$\alpha_{z}$ is the phase angle between the wave and force.

Finally, it should be noted that the computations of the above coefficients in (25) can be found in [38].

6.2. Dynamic Model of the Tensegrity-Based Water Wave Energy Harvester. As stated in Section 2, the harvester has three degrees of freedom. Therefore, three generalized coordinates, chosen as $\mathbf{q}=\left[\begin{array}{lll}q_{1} & q_{2} & q_{3}\end{array}\right]^{T}=\left[\begin{array}{lll}y & z & \theta\end{array}\right]^{T}$, are needed to develop the dynamic model.

In order to derive an appropriate dynamic model of the harvester, the following hypotheses are made:

(i) The links of the mechanism, except for the float, are massless.

(ii) The springs are massless.

(iii) There is no friction in the harvester's revolute, prismatic, and spherical joints.

The equations of motion of the harvester are developed using the Lagrangian approach; namely,

$$
\frac{d}{d t} \frac{\partial T}{\partial \dot{\mathbf{q}}}-\frac{\partial T}{\partial \mathbf{q}}+\frac{\partial E}{\partial \mathbf{q}}=\mathbf{Q}_{k},
$$

where $T$ and $E$ are the kinetic and potential energies of the harvester and $\mathbf{Q}_{k}$ is the vector of nonconservative forces acting on the system. In [37], the translation of the float along $Y$ axis is defined as surge, the translation of the float along $Z$ axis is defined as heave, and the rotation of the float with respect to $X$ axis is defined as pitch. The kinetic energy, due only to the surge, heave, and pitch movements of the float, can be expressed as

$$
\mathbf{T}=\frac{1}{2} \dot{\mathbf{q}}^{T} \mathbf{M} \dot{\mathbf{q}}
$$

where

$$
\mathbf{M}=\left[\begin{array}{ccc}
m+a_{w y} & 0 & 0 \\
0 & m+a_{w y} & 0 \\
0 & 0 & I_{y}+A_{w}
\end{array}\right]
$$

$I_{y}$ is the mass moment of inertia with respect to $Y$ axis and $A_{w}$ is added-mass moment of inertia due to pitching. The potential energies due to heaving and pitching motions of the top platform are described by McCormick [37] as

$$
\begin{aligned}
& E_{p z}=\frac{1}{2} \rho g A_{w p} q_{2}^{2}, \\
& E_{p \theta}=\frac{1}{2} C q_{3}^{2},
\end{aligned}
$$

where $C$ is the restoring moment constant, defined for a bottom-flat body in terms of the draft. The total potential energy of the harvester becomes

$$
\begin{aligned}
E= & U+E_{p z}+E_{p \theta} \\
= & \frac{1}{2} \rho g A_{w p} q_{2}^{2}+\frac{1}{2} C q_{3}^{2}+K\left[\sqrt{\sigma_{1}^{2}+\sigma_{2}^{2}}-l_{0}\right]^{2} \\
& +K\left[\sqrt{\sigma_{3}^{2}+\sigma_{4}^{2}}-l_{0}\right]^{2} .
\end{aligned}
$$

The nonconservative forces, which correspond to the radiation damping force, viscous damping force, and water wave induced forces, can be expressed as

$$
\mathbf{Q}_{k}=\left[\begin{array}{c}
-b_{v y} \dot{q}_{1} \\
F_{z 0} \cos (\omega t)-\left(b_{r z}+b_{v z}\right) \dot{q}_{2} \\
M_{\theta 0} \cos (\omega t)-b_{r \theta} \dot{q}_{3}
\end{array}\right] .
$$


Substituting (27), (30), and (31) into (26), the dynamic model of the harvester can be rewritten as

$$
\mathbf{M} \ddot{\mathbf{q}}+\mathbf{B} \dot{\mathbf{q}}+\mathbf{G}=\mathbf{F},
$$

where

$$
\begin{aligned}
\mathbf{B} & =\left[\begin{array}{ccc}
b_{v y} & 0 & 0 \\
0 & b_{r z}+b_{v z}+b_{p z} & 0 \\
0 & 0 & b_{r \theta}
\end{array}\right], \\
\mathbf{G} & =\left[\begin{array}{lll}
\frac{\partial E}{\partial q_{1}} & \frac{\partial E}{\partial q_{2}} & \frac{\partial E}{\partial q_{3}}
\end{array}\right]^{T}, \\
\mathbf{F} & =\left[\begin{array}{llll}
0 & F_{z o} \cos \omega t & M_{\theta 0} \sin \omega t
\end{array}\right]^{T} .
\end{aligned}
$$

The elements of $\mathbf{G}$ are detailed in the Appendix. For (32), it should be noted that $b_{v y}$ is viscous damping coefficient corresponding to the surge movements of the float while $b_{r \theta}$ is the radiation damping coefficient due to pitching motion. $M_{\theta 0}$ is the water-induced torque amplitude (applied on the float). The computations of $b_{v y}, b_{r \theta}, M_{\theta 0}$, and $C$ can also be found in [37]. These computations are also not repeated here.

\section{Energy Harvesting}

In this section, two energy harvesting systems are researched, respectively. One is a conventional heaving system and the other is the tensegrity-based water wave harvester. Also, the powers of the two systems have been computed, respectively. The parameters of water waves are selected as $H=0.2 \mathrm{~m}$, $T=6 \mathrm{~s}$, and $h=100 \mathrm{~m} . H$ is the wave height measured from the trough to the crest while $T$ is the wave period. $h$ denotes the water depth. Moreover, the floats used in the two energy harvesting systems are supposed to have the same dimensions as $L=1 \mathrm{~m}, D=0.1 \mathrm{~m}$, and $d=0.05 \mathrm{~m}$.

7.1. Conventional Heaving System. For a conventional heaving system, the motion of the float is expressed by (25). For the given water wave parameters and the dimensions of the float, the coefficients of (25) can be calculated according to [38]. The results are listed in Table 1.

Solving (25) yields the position and velocity of the float which are shown in Figure 5. The power of the heaving body is given by [37]

$$
P_{z}(t)=F_{z}(t) \frac{d z(t)}{d t},
$$

where $F_{z}(t)$ is the wave introduced heaving force on the float.

The power for take-off, $P(t)$, is given by the difference between the available power $\left(P_{z}(t)\right)$ and the power dissipated due to radiation $\left(P_{r z}(t)\right)$ and viscous effects $\left(P_{v z}(t)\right)$ :

$$
P(t)=P_{z}(t)-P_{r z}(t)-P_{v z}(t) .
$$

The average power for take-off over one period of time is given by

$$
P_{\mathrm{ave}}=\frac{1}{T} \int_{T} P(t) d t
$$

TABLE 1: Conventional heaving float coefficients.

\begin{tabular}{lcc}
\hline Coefficient & Value & Unit \\
\hline$m$ & 51.50 & $\mathrm{~kg}$ \\
$a_{w z}$ & 454.19 & $\mathrm{~kg}$ \\
$b_{r z}$ & 1065.50 & $\mathrm{~N} \cdot \mathrm{s} / \mathrm{m}$ \\
$b_{v z}$ & 114.91 & $\mathrm{~N} \cdot \mathrm{s} / \mathrm{m}$ \\
$b_{p z}$ & 0 & $\mathrm{~N} \cdot \mathrm{s} / \mathrm{m}$ \\
$A_{w p}$ & 1 & $\mathrm{~m}$ \\
$N$ & 0 & - \\
$k_{s}$ & 0 & $\mathrm{~N} / \mathrm{m}$ \\
$F_{z 0}$ & 2012.10 & $\mathrm{~N}$ \\
$\alpha_{z}$ & 0 & $\mathrm{rad}$ \\
$\omega_{n z}$ & 4.47 & $\mathrm{Rad} / \mathrm{s}$ \\
$b_{c z}$ & 4518.60 & $\mathrm{~N} \cdot \mathrm{s} / \mathrm{m}$ \\
$Z_{0}$ & 0.21 & $\mathrm{~m}$ \\
\hline
\end{tabular}

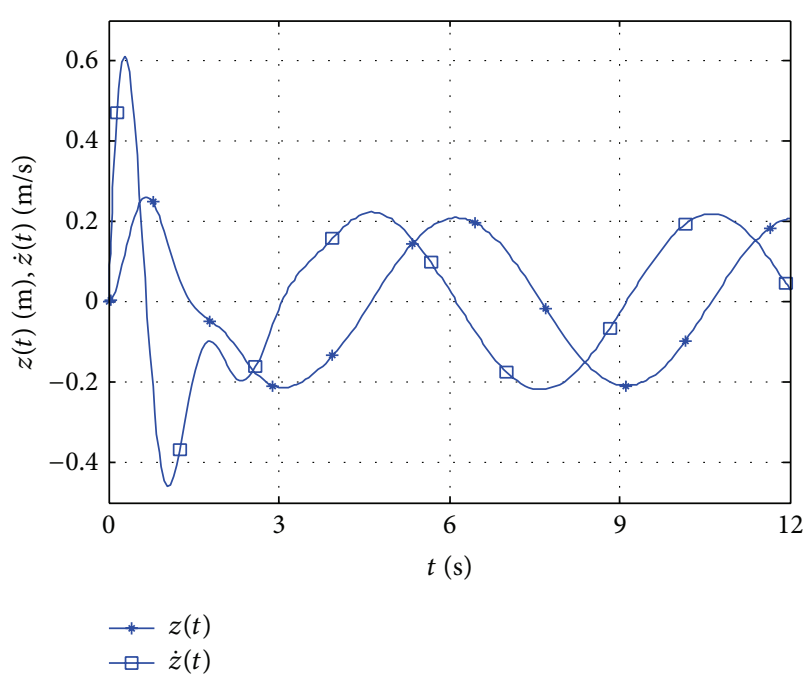

Figure 5: Motion of the conventional heaving system.

The water wave energy and power are [37]

$$
\begin{aligned}
& E=\frac{\rho g^{2} H^{2} T^{2} b}{16 \pi}, \\
& \mathbf{P}=\frac{\rho g^{2} H^{2} T b}{32 \pi} \mathbf{i} .
\end{aligned}
$$

Applying (36) over two wave periods of the function shown in Figure 6 gives an average power $P_{\text {ave }}=0.154 \mathrm{~kW}$. Since the float's breadth is $1 \mathrm{~m}$, then we can compare this result with the power contained in one meter of wave front. The maximum available power per meter of wave front is $P=0.236 \mathrm{~kW}$ (computed by (38)). Therefore, $65.17 \%$ of the wave energy can be harvested with electrical generators.

7.2. Tensegrity-Based Wave Energy Harvester. For the harvester considered here, it has infinitesimal mechanisms inherent of many tensegrity systems. This means that there are infinitesimal deformations of the mechanism that do not require any changes in the lengths of the harvester's 
TABLE 2: Tensegrity-based harvester coefficients.

\begin{tabular}{lcc}
\hline Coefficient & Value & Unit \\
\hline$m$ & 51.50 & $\mathrm{~kg}$ \\
$a_{w y}$ & 2.02 & $\mathrm{~kg}$ \\
$a_{w z}$ & 454.19 & $\mathrm{~kg}$ \\
$A_{w}$ & 15.03 & $\mathrm{~kg} \cdot \mathrm{m}^{2}$ \\
$I_{x}$ & 4.33 & $\mathrm{Kg} \cdot \mathrm{m}^{2}$ \\
$C$ & 849.58 & $\mathrm{~N} \cdot \mathrm{m} / \mathrm{rad}$ \\
$b_{r z}$ & 1065.50 & $\mathrm{~N} \cdot \mathrm{s} / \mathrm{m}$ \\
$b_{r \theta}$ & 88.79 & $\mathrm{~N} \cdot \mathrm{m} \cdot \mathrm{s} / \mathrm{rad}$ \\
$b_{v y}$ & 114.91 & $\mathrm{~N} \cdot \mathrm{s} / \mathrm{m}$ \\
$b_{v z}$ & 114.91 & $\mathrm{~N} \cdot \mathrm{s} / \mathrm{m}$ \\
$b_{p z}$ & 0 & $\mathrm{~N} \cdot \mathrm{s} / \mathrm{m}$ \\
$A_{w p}$ & 1 & $\mathrm{M}$ \\
$F_{z 0}$ & 2012.10 & $\mathrm{~N}$ \\
$M_{\theta 0}$ & 18.77 & $\mathrm{~N} \cdot \mathrm{m}$ \\
$\alpha_{z}$ & 0 & $\mathrm{rad}$ \\
\hline
\end{tabular}

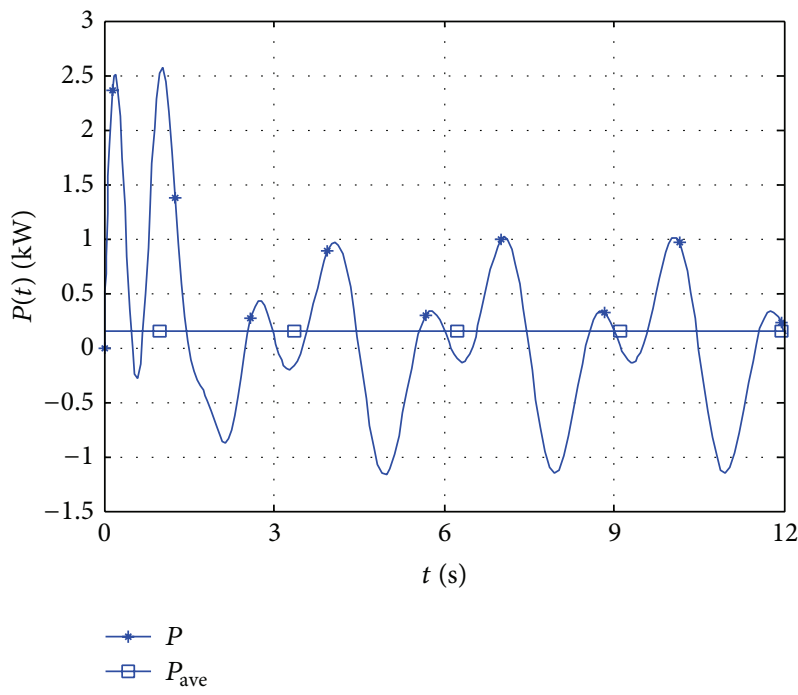

Figure 6: Power for take-off of the conventional heaving system.

components. It follows that some wave energy would not be harvested as the mechanism could deform some degree without the deformation being felt by the linear generators. However, since the deformations are infinitesimal, the effects of infinitesimal mechanisms are negligible.

Let the dimensions of the float in the tensegrity-based water wave harvester be the same as the conventional heaving float. The additional constant physical parameters are $L_{0}=$ $4 \mathrm{~m}$ and $K=10 \mathrm{~N} / \mathrm{m}$. Table 2 contains the values of the coefficients (computed according to [38]) for the equation of motion (see (32)).

The simulation is performed over two wave periods, that is, 12 seconds. Figures 7-9 show the position and velocity response of the float: surge, heave, and pitch.

Figure 10 shows the instantaneous power for take-off. The average power over two wave periods is $P_{\text {ave }}=0.186 \mathrm{~kW}$. The

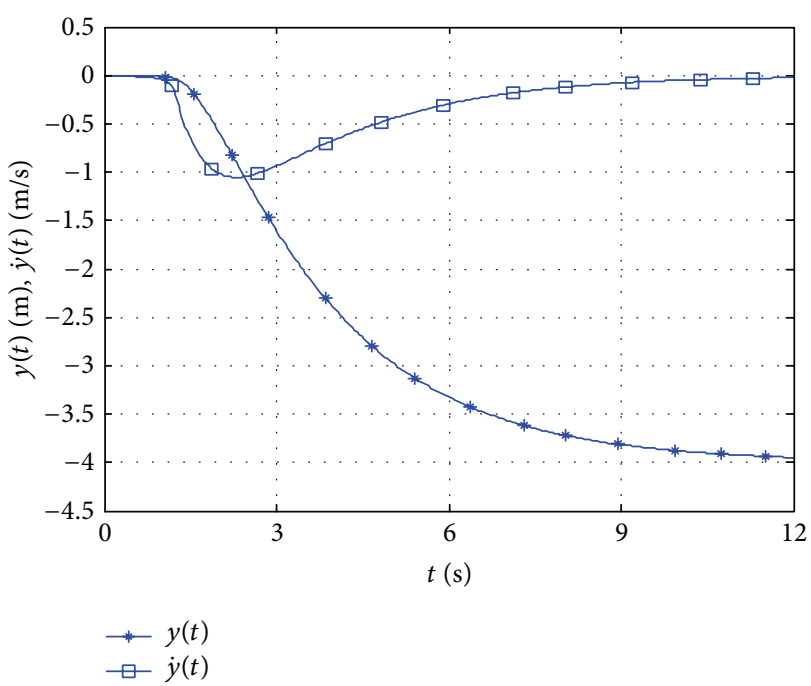

FIGURE 7: Surge motions of the tensegrity-based water wave energy harvester.

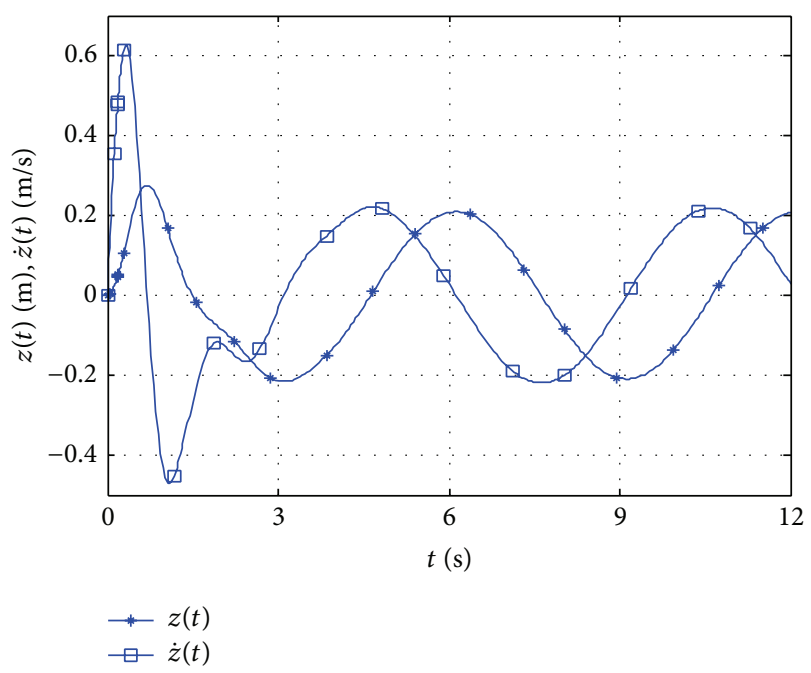

FIGURE 8: Heave motions of the tensegrity-based water wave energy harvester.

power contained in one meter of wave front is $P=0.236 \mathrm{~kW}$ (computed by (38)). Therefore, $78.76 \%$ of the available energy could be harvested by electrical generators. By comparing Figures 6 with 10, it is found that the proposed tensegritybased harvester allows harvesting $13.59 \%$ more energy than a conventional heaving device under linear water wave conditions. For the conventional heaving device, the movement of the float is translation along the $Z$ axis. It is proper to say that the conventional heaving device has one degree of freedom. However, the possible movements of the proposed harvester are rotations about the $X$ axis and translations along the $Y$ and $Z$ axes (see Section 2). It is thus proper to say that the proposed harvester has three degrees of freedom. That is why the harvester can harvest more energy than a conventional device. 


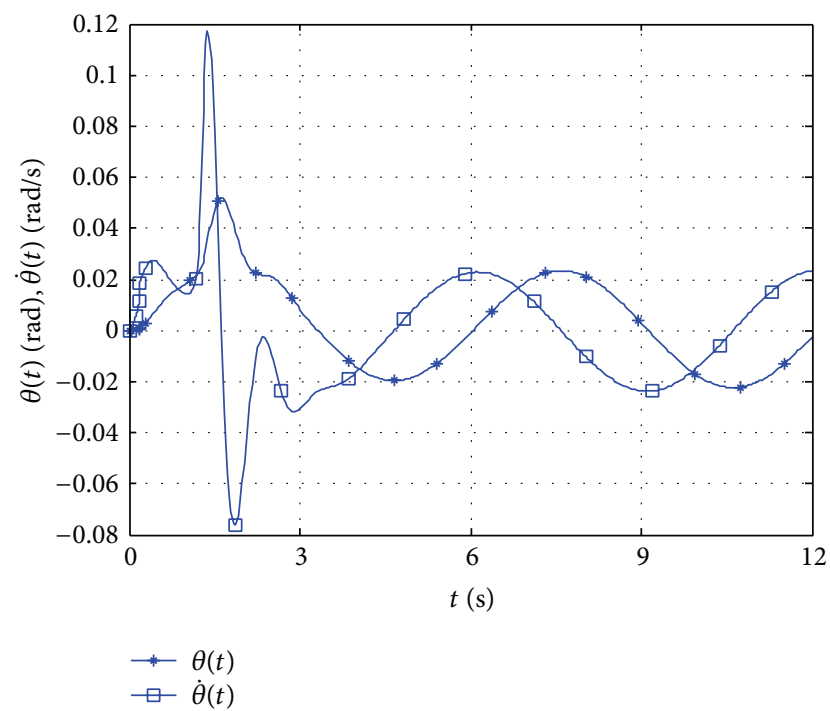

FIGURE 9: Pitch motions of the tensegrity-based water wave energy harvester.

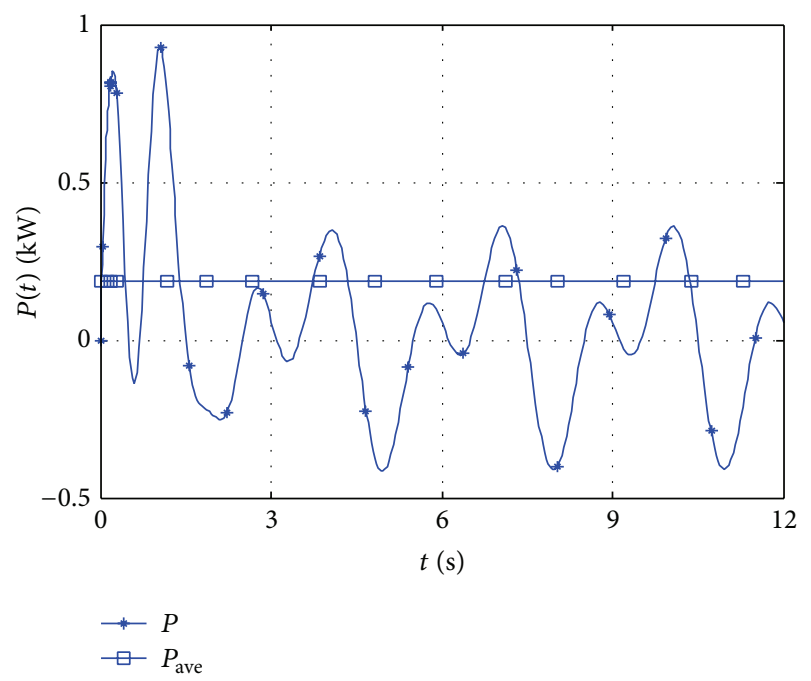

FIGURE 10: Tensegrity-based harvester: power for take-off.

\section{Conclusion}

A tensegrity-based water wave energy harvester was proposed in this work. The geometry of the harvester was described. The solutions to the direct and inverse kinematic problems were found by using a geometric method. The Jacobian matrix and singular configurations were subsequently computed. Then, the input and output workspaces were computed on the basis of the analysis of the obtained singular configurations. Afterwards, the dynamic analysis was performed considering the interaction with linear water waves, considering added mass, radiation damping, and viscous damping phenomena. It was shown that the proposed tensegrity-based water wave energy harvester allows harvesting $13.59 \%$ more energy than a conventional heaving system.

\section{Appendix}

\section{Elements of G}

The elements of $\mathbf{G}$ in (32) are as follows:

$$
\begin{aligned}
& G_{1}=\frac{\partial E}{\partial q_{1}}=2 K\{1 \\
& -L_{0}\left[\left(q_{1}-\frac{L \cos q_{3}}{2}-\frac{D \sin q_{3}}{2}+\frac{L}{2}\right)^{2}\right. \\
& \left.\left.+\left(q_{2}-\frac{D \cos q_{3}}{2}+\frac{L \sin q_{3}}{2}\right)^{2}\right]^{-1 / 2}\right\} \cdot\left(q_{1}\right. \\
& \left.-\frac{L \cos q_{3}}{2}-\frac{D \sin q_{3}}{2}+\frac{L}{2}\right)+2 K\left(q_{1}+\frac{L \cos q_{3}}{2}\right. \\
& \left.-\frac{D \sin q_{3}}{2}-\frac{L}{2}\right) \cdot\{1 \\
& -L_{0}\left[\left(q_{1}+\frac{L \cos q_{3}}{2}-\frac{D \sin q_{3}}{2}-\frac{L}{2}\right)^{2}\right. \\
& \left.\left.+\left(q_{2}-\frac{D \cos q_{3}}{2}-\frac{L \sin q_{3}}{2}\right)^{2}\right]^{-1 / 2}\right\} \text {, } \\
& G_{2}=\frac{\partial E}{\partial q_{2}}=\rho g A_{w p} q_{2}+2 K\left(q_{2}-\frac{D \cos q_{3}}{2}\right. \\
& \left.+\frac{L \sin q_{3}}{2}\right) \cdot\{1 \\
& -L_{0}\left[\left(q_{1}-\frac{L \cos q_{3}}{2}-\frac{D \sin q_{3}}{2}+\frac{L}{2}\right)^{2}\right. \\
& \left.\left.+\left(q_{2}-\frac{D \cos q_{3}}{2}+\frac{L \sin q_{3}}{2}\right)^{2}\right]^{-1 / 2}\right\}+2 K\{1 \\
& -L_{0}\left[\left(q_{1}+\frac{L \cos q_{3}}{2}-\frac{D \sin q_{3}}{2}-\frac{L}{2}\right)^{2}\right. \\
& \left.\left.+\left(q_{2}-\frac{D \cos q_{3}}{2}-\frac{L \sin q_{3}}{2}\right)^{2}\right]^{-1 / 2}\right\} \cdot\left(q_{2}\right. \\
& \left.-\frac{D \cos q_{3}}{2}-\frac{L \sin q_{3}}{2}\right) \\
& G_{3}=\frac{\partial E}{\partial q_{3}}=C q_{3}+2 K\{1 \\
& -L_{0}\left[\left(q_{1}-\frac{L \cos q_{3}}{2}-\frac{D \sin q_{3}}{2}+\frac{L}{2}\right)^{2}\right. \\
& \left.\left.+\left(q_{2}-\frac{D \cos q_{3}}{2}+\frac{L \sin q_{3}}{2}\right)^{2}\right]^{-1 / 2}\right\} \cdot\left[\left(q_{1}\right.\right.
\end{aligned}
$$




$$
\begin{aligned}
& \left.-\frac{L \cos q_{3}}{2}-\frac{D \sin q_{3}}{2}+\frac{L}{2}\right)\left(\frac{L \sin q_{3}}{2}\right. \\
& \left.-\frac{D \cos q_{3}}{2}\right)+\left(q_{2}-\frac{D \cos q_{3}}{2}+\frac{L \sin q_{3}}{2}\right) \\
& \left.\cdot\left(\frac{D \sin q_{3}}{2}+\frac{L \cos q_{3}}{2}\right)\right]+2 K\{1 \\
& -L_{0}\left[\left(q_{1}+\frac{L \cos q_{3}}{2}-\frac{D \sin q_{3}}{2}-\frac{L}{2}\right)^{2}\right. \\
& \left.\left.+\left(q_{2}-\frac{D \cos q_{3}}{2}-\frac{L \sin q_{3}}{2}\right)^{2}\right]^{-1 / 2}\right\} \cdot\left[-\left(q_{1}\right.\right. \\
& \left.+\frac{L \cos q_{3}}{2}-\frac{D \sin q_{3}}{2}-\frac{L}{2}\right)\left(\frac{L \sin q_{3}}{2}\right. \\
& \left.+\frac{D \cos q_{3}}{2}\right)+\left(q_{2}-\frac{D \cos q_{3}}{2}-\frac{L \sin q_{3}}{2}\right) \\
& \left.+\left(\frac{D \sin q_{3}}{2}-\frac{L \cos q_{3}}{2}\right)\right] \cdot
\end{aligned}
$$

\section{Competing Interests}

The authors declare that they have no competing interests.

\section{Acknowledgments}

This research is supported by the National Natural Science Foundation of China (no. 51375360).

\section{References}

[1] R. Motro, Tensegrity: Structural Systems for the Future, Kogen Page Science, Guildford, UK, 2003.

[2] R. E. Skelton and M. C. Oliveira, Tensegrity Systems, Springer, New York, NY, USA, 2009.

[3] C. R. Calladine, "Buckminster Fuller's 'Tensegrity' structures and Clerk Maxwell's rules for the construction of stiff frames," International Journal of Solids and Structures, vol. 14, no. 2, pp. 161-172, 1978.

[4] S. Pellegrino, "A class of tensegrity domes," International Journal of Space Structures, vol. 7, no. 2, pp. 127-142, 1992.

[5] F. Fu, "Structural behavior and design methods of Tensegrity domes," Journal of Constructional Steel Research, vol. 61, no. 1, pp. 23-25, 2005.

[6] L. Rhode-Barbarigos, N. B. Hadj Ali, R. Motro, and I. F. C. Smith, "Designing tensegrity modules for pedestrian bridges," Engineering Structures, vol. 32, no. 4, pp. 1158-1167, 2010.

[7] L. Rhode-Barbarigos, N. B. H. Ali, R. Motro, and I. F. C. Smith, "Design aspects of a deployable tensegrity-hollow-rope footbridge," International Journal of Space Structures, vol. 27, no. 2-3, pp. 81-95, 2012.

[8] S. Korkmaz, N. B. H. Ali, and I. F. C. Smith, "Configuration of control system for damage tolerance of a tensegrity bridge," Advanced Engineering Informatics, vol. 26, no. 1, pp. 145-155, 2012.
[9] R. E. Skelton, F. Fraternali, G. Carpentieri, and A. Micheletti, "Minimum mass design of tensegrity bridges with parametric architecture and multiscale complexity," Mechanics Research Communications, vol. 58, pp. 124-132, 2014.

[10] J. Duffy, J. Rooney, B. Knight, and C. D. Crane III, "Review of a family of self-deploying tensegrity structures with elastic ties," Shock and Vibration Digest, vol. 32, no. 2, pp. 100-106, 2000.

[11] A. Hanaor, "Double-layer tensegrity grids as deployable structures," International Journal of Space Structures, vol. 8, no. 1-2, pp. 135-143, 1993.

[12] G. Tibert, Deployable tensegrity structures in space applications [Ph.D. thesis], Royal Institute of Technology, Stockholm, Sweden, 2002.

[13] N. Fazli and A. Abedian, "Design of tensegrity structures for supporting deployable mesh antennas," Scientia Iranica, vol. 18, no. 5, pp. 1078-1087, 2011.

[14] N. Vassart and R. Motro, "Multiparametered form finding method: application to tensegrity systems," International Journal of Space Structures, vol. 14, no. 2, pp. 147-154, 1999.

[15] K. Koohestani, "Form-finding of tensegrity structures via genetic algorithm," International Journal of Solids and Structures, vol. 49, no. 5, pp. 739-747, 2012.

[16] A. G. Tibert and S. Pellegrino, "Review of form-finding methods for tensegrity structures," International Journal of Space Structures, vol. 18, no. 4, pp. 209-223, 2003.

[17] S. H. Juan and J. M. M. Tur, "Tensegrity frameworks: static analysis review," Mechanism and Machine Theory, vol. 43, no. 7, pp. 859-881, 2008.

[18] D. E. Ingber, "Cellular tensegrity: defining new rules of biological design that govern the cytoskeleton," Journal of Cell Science, vol. 104 , no. 3, pp. 613-627, 1993.

[19] D. E. Ingber, "The architecture of life," Scientific American, vol. 278, no. 1, pp. 48-57, 1998.

[20] D. E. Ingber, “Tensegrity I. Cell structure and hierarchical systems biology," Journal of Cell Science, vol. 116, no. 7, pp. 1157$1173,2003$.

[21] S. M. Levin, "The tensegrity-truss as a model for spinal mechanics: biotensegrity," Journal of Mechanics in Medicine and Biology, vol. 2, no. 3, pp. 375-388, 2002.

[22] I. J. Oppenheim and W. O. Williams, "Tensegrity prisms as adaptive structures," in Proceedings of the ASME International Mechanical Engineering Congress and Exposition, Dallas, Tex, USA, November 1997.

[23] C. Sultan, M. Corless, and R. E. Skelton, "Tensegrity flight simulator," Journal of Guidance, Control, and Dynamics, vol. 23, no. 6, pp. 1055-1064, 2000.

[24] C. Sultan, M. Corless, and R. E. Skelton, "Peak-to-peak control of an adaptive tensegrity space telescope," in Smart Structures and Materials 1999: Mathematics and Control in Smart Structures, vol. 3667 of Proceedings of SPIE, pp. 190-201, The International Society for Optical Engineering, Newport Beach, Calif, USA, March 1999.

[25] C. Paul, F. J. Valero-Cuevas, and H. Lipson, "Design and control of tensegrity robots for locomotion," IEEE Transactions on Robotics, vol. 22, no. 5, pp. 944-957, 2006.

[26] A. G. Rovira and J. M. M. Tur, "Control and simulation of a tensegrity-based mobile robot," Robotics and Autonomous Systems, vol. 57, no. 5, pp. 526-535, 2009.

[27] S. Hirai and R. Imuta, "Dynamic simulation of six-strut tensegrity rolling," in Proceedings of the 2012 IEEE International Conference on Robotics and Biomimetics, Guangzhou, China, 2012. 
[28] M. Q. Marshall, Analysis of tensegrity-based parallel platform devices [M.S. thesis], University of Florida, Gainesville, Fla, USA, 2003.

[29] S. M. M. Shekarforoush, M. Eghtesad, and M. Farid, "Kinematic and static analyses of statically balanced spatial tensegrity mechanism with active compliant components," Journal of Intelligent \& Robotic Systems, vol. 71, no. 3-4, pp. 287-302, 2013.

[30] C. D. Crane III, J. Bayat, V. Vikas, and R. Roberts, "Kinematic analysis of a planar tensegrity mechanism with pre-stressed springs," in Advances in Robot Kinematics: Analysis and Design, J. Lenarčič and P. Wenger, Eds., pp. 419-427, Springer, Batz-surMer, France, 2008.

[31] J. M. McCarthy, "21st century kinematics: synthesis, compliance, and tensegrity," Journal of Mechanisms and Robotics, vol. 3, no. 2, Article ID 020201, 2011.

[32] J. T. Scruggs and R. E. Skelton, "Regenerative tensegrity structures for energy harvesting applications," in Proceedings of the 45th IEEE Conference on Decision and Control (CDC '06), pp. 2282-2287, IEEE, San Diego, Calif, USA, December 2006.

[33] M. R. Sunny, C. Sultan, and R. K. Kapania, "Optimal energy harvesting from a membrane attached to a tensegrity structure," AIAA Journal, vol. 52, no. 2, pp. 307-319, 2014.

[34] R. E. Vasquez, C. D. Crane III, and J. C. Correa, "Analysis of a planar tensegrity mechanism for ocean wave energy harvesting," Journal of Mechanisms and Robotics, vol. 6, no. 3, Article ID 031015, 2014.

[35] S. V. Sreenivasan, K. J. Waldron, and P. Nanua, "Closedform direct displacement analysis of a 6-6 Stewart platform," Mechanism and Machine Theory, vol. 29, no. 6, pp. 855-864, 1994.

[36] C. D. Crane III and J. Duffy, Kinematic Analysis of Robot Manipulators, Cambridge University Press, New York, NY, USA, 1998.

[37] M. McCormick, Ocean Wave Energy Conversion, Dover, Mineola, NY, USA, 2007.

[38] R. E. Vasquez, Analysis of a tensegrity system for ocean wave energy harvesting [Ph.D. thesis], University of Florida, Gainesville, Fla, USA, 2011. 


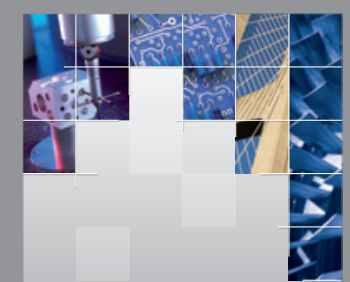

\section{Enfincering}
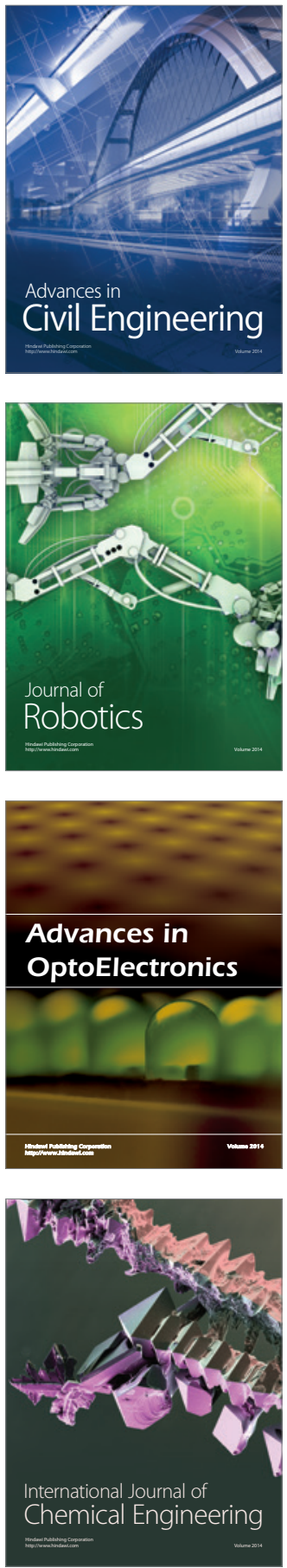

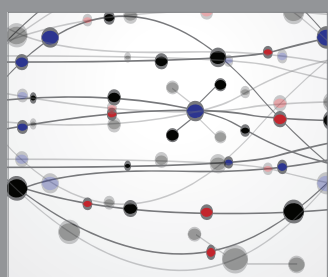

The Scientific World Journal

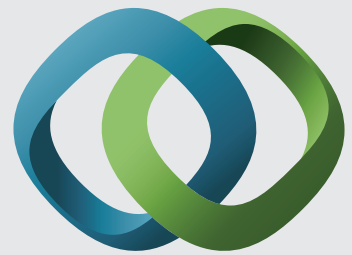

\section{Hindawi}

Submit your manuscripts at

http://www.hindawi.com
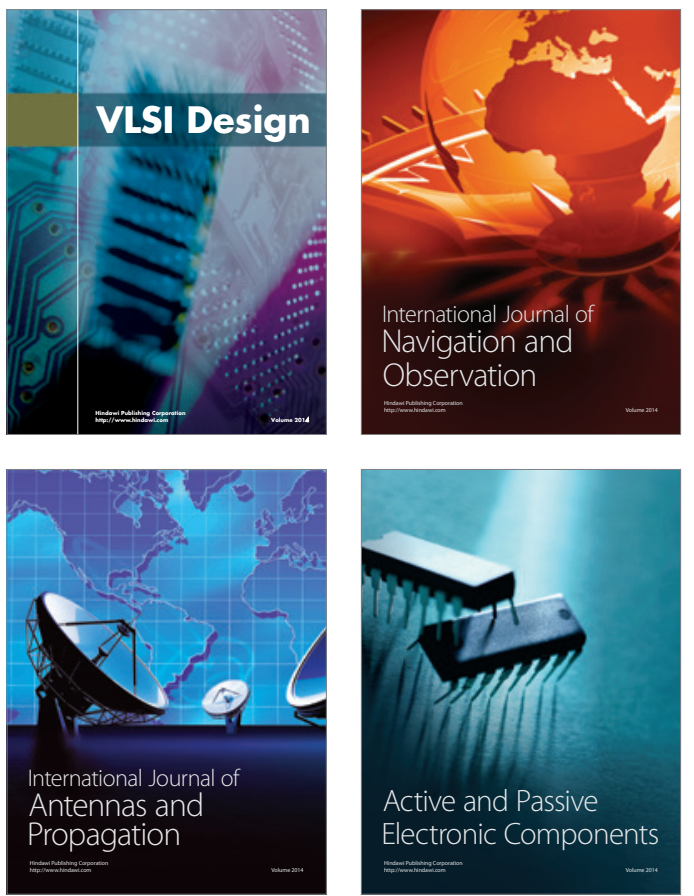
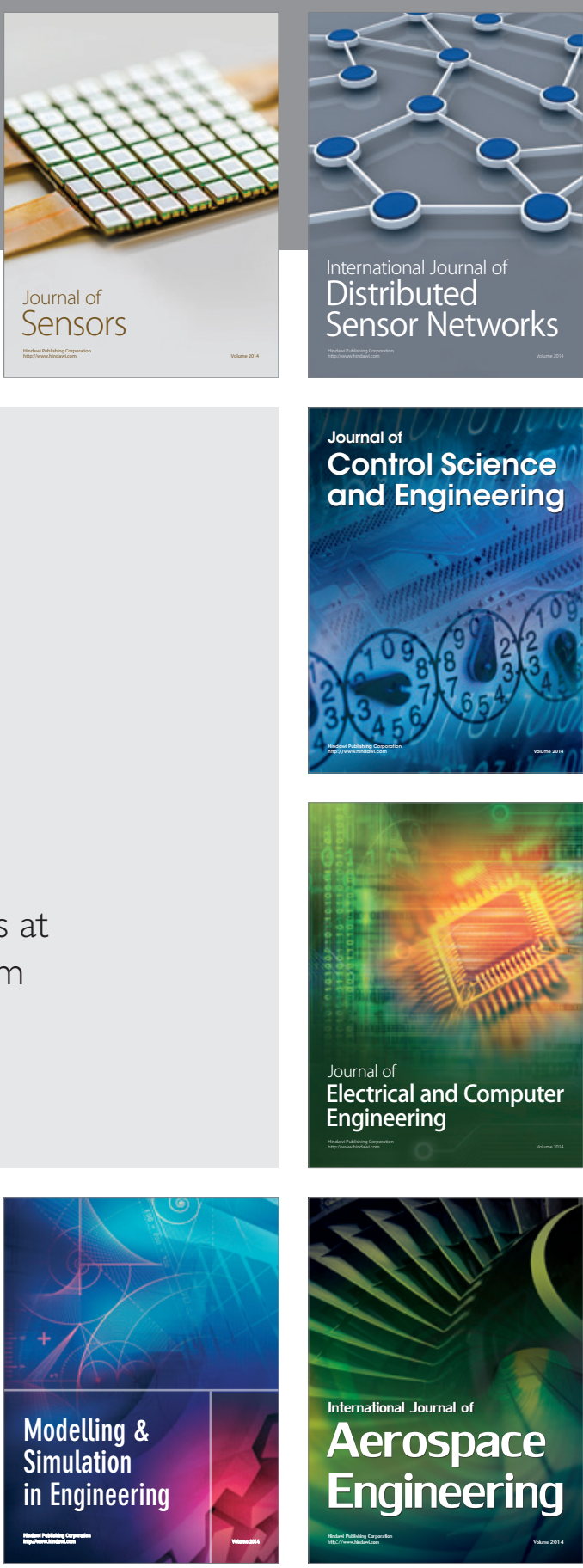

International Journal of

Distributed

Sensor Networks

Journal of

Control Science

and Engineering
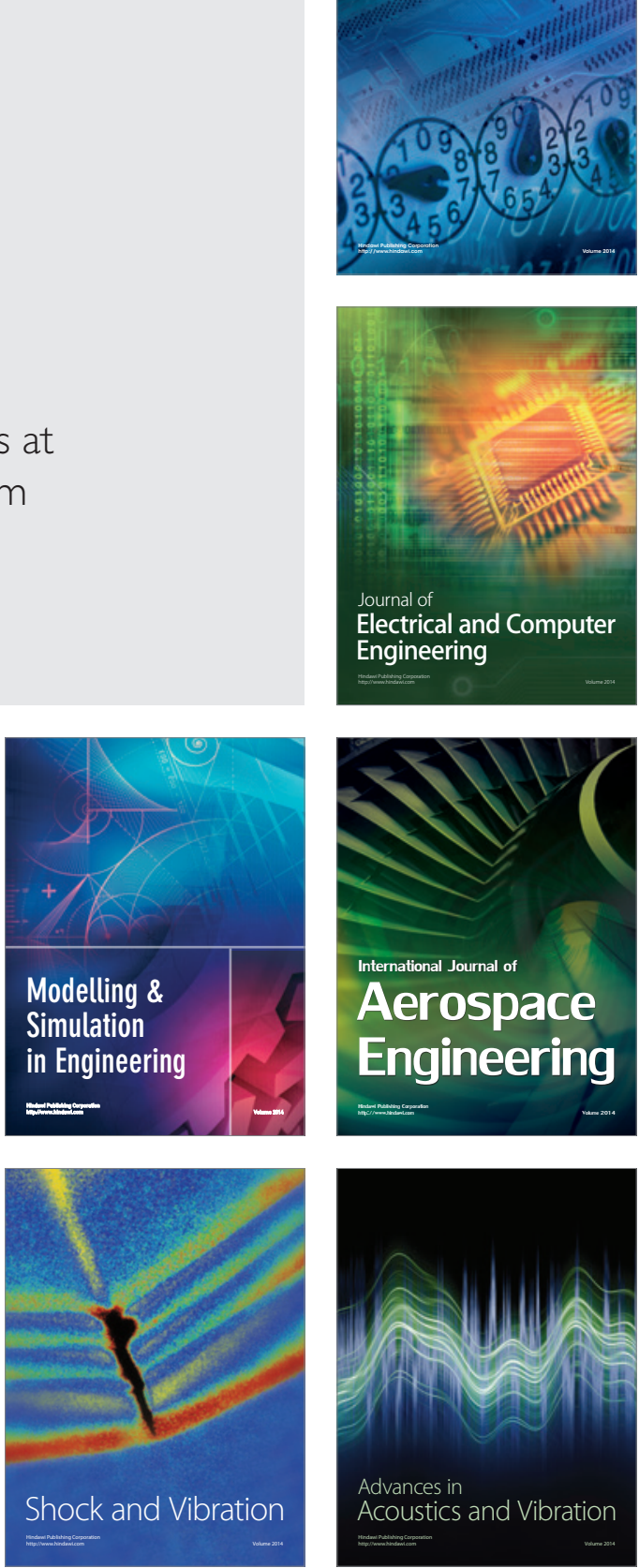